Published in "Soft Matter 4: 952-961, 2008"

which should be cited to refer to this work.

\title{
Supramolecular routes towards liquid crystalline side-chain polymers
}

\author{
Matthew R. Hammond ${ }^{a}$ and Raffaele Mezzenga ${ }^{* a b}$
}

Supramolecular attachment of mesogenic or non-mesogenic side chains to polymer backbones can result in the formation of liquid crystalline morphologies. The various parameters that can be tuned in order to achieve these morphologies, such as the type of non-covalent bonding chemistry (hydrogen bonding, ionic bonding, metal coordination, $\pi-\pi$ interactions), the polymeric template architecture, and the side chain structure and properties, are reviewed in what follows, with emphasis placed on the role these parameters play in the determination of the final material morphologies and properties.

\section{Introduction}

Given its versatility, power, and potentially facile applicability, supramolecular chemistry has gained remarkable attention during the last twenty years as a viable technique to design complex self-assembled systems based on non-covalent bonds. ${ }^{1,2}$ The list of potential benefits from the incorporation of supramolecular chemistry concepts into polymer science is extremely long, and constantly growing. Improved polymer processing and mechanical properties, blend compatibilization, the facile incorporation of functional groups, and new routes to the creation of mesostructured self-organizing materials are only the tip of the proverbial iceberg. The topic of this review is centered on the use of competing interactions ${ }^{3}$ to design mesomorphic polymeric structures, whose periodicities typically span the $\sim$ nm length scale. This is achieved in supramolecular macromolecules by non-covalently coupling otherwise incompatible components. Because by this route nm-spaced interfaces are introduced in the materials, the resulting supramolecularly structured objects bear many similarities with more conventional

${ }^{a}$ Department of Physics and Fribourg Center for Nanomaterials, University of Fribourg, Chemin du Musée 3, 1700 Fribourg, Switzerland. E-mail. raffaele.mezzenga@unifr.ch; Fax: +41 (0)26 3009747

${ }^{b}$ Nestlé Research Center, Vers-chez-les-blanc, 1000 Lausanne 26, Switzerland liquid crystalline polymers. Mesomorphism is also well known in microphase-separating block or graft copolymers, where incompatible blocks are forced to reside at the interface defined by the covalent block junction. The final packing symmetry of the resulting mesophase is determined by the curvature of the interface (which is governed by a balance between immiscibility and chain stretching effects). ${ }^{4}$ Supramolecular chemistry thus offers another, perhaps simpler route to the creation of such interfaces, and indeed, in recent years, telechelic block copolymers have started to be designed based on supramolecular approaches. ${ }^{5-7}$ Furthermore, the supramolecular route also holds potential advantages with respect to the problems of "topological dereliction", i.e. the tendency to become trapped in local free energy minima along the path to the desired morphology, often encountered in self-organizing polymeric systems. ${ }^{3}$ By reducing the interaction strengths that lead to interface creation (i.e. noncovalent vs. covalent bonds), a smoother free energy pathway to the desired morphology may result. The last important feature specific to supramolecularly self-assembled mesomorphic materials is the possible reversible nature of binding, and thus, of interface creation, which can result in unique properties. ${ }^{8}$

The nature of the non-covalent bonding method (or methods) chosen for a given supramolecular synthesis will obviously have a profound effect on the resulting material properties. The bonding types most commonly employed to create solid supramolecular materials consist of electrostatic interactions between 

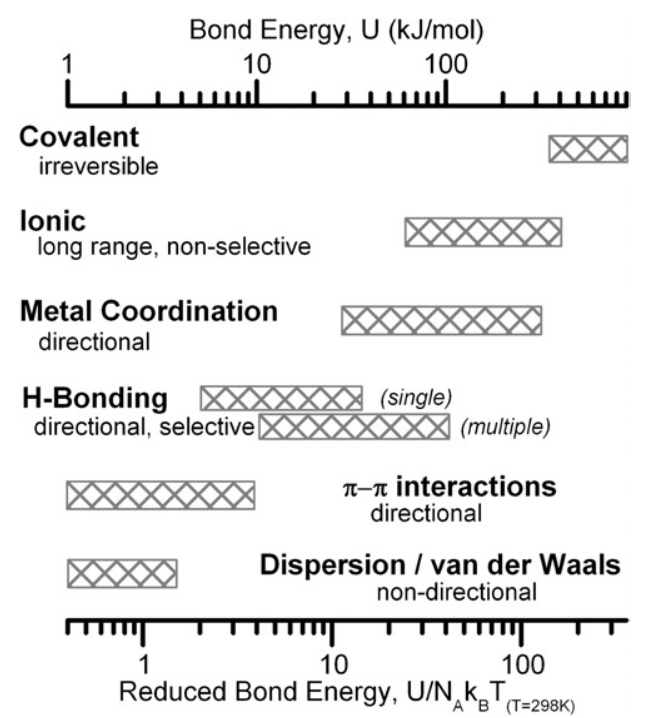

Fig. 1 Bond energies and relevant characteristics for different bonding interactions.

charged species ("ionic bonding"), metal-ligand coordination, hydrogen bonding, $\pi-\pi$ interactions, and dispersion/van der Waals forces. Fig. 1 shows approximate bond energies (both in absolute terms and relative to $k T$ ) and other relevant information for the different types of bonds considered here.

In this review, we provide illustrative examples of mesomorphous supramolecular materials based on macromolecular templates and assembled via the various non-covalent forces. Supramolecular polymers self-assembled via non-covalent interactions between low molecular weight entities, although often mesomorphous, are largely excluded from consideration here (for reviews, see ref. 9-11). Block copolymers created by bonding between telechelic polymers are also left out (see ref. 5,7,12). Particular emphasis is placed on materials showing topological mesomorphism (ordering in the spatial position of structural units) in the solid state, although some materials showing nematic behavior (ordering in the orientation of structural units) will also be mentioned. As such, most of the structures described herein have been elucidated primarily via small- and/or wideangle X-ray scattering (SAXS/WAXS) and optical birefringence measurements, as well as, in some cases, with transmission electron microscopy (TEM) or dynamic rheology. Complex formation itself is studied primarily with FTIR, NMR, and elemental analysis.

The general issues we wish to address presently are sketched in Fig. 2. Firstly, what follows is organized according to the type of supramolecular bonding interaction. With each complexation method come various advantages as well as limitations, as will become apparent. In addition, we provide some examples wherein incorporation of multiple bonding pathways can leverage the advantages and circumvent the limitations of any single type of interaction alone. Secondly, we wish to touch upon the different types of low molecular weight building blocks, or "tectons", that can be "grafted" to macromolecular templates in order to impart specific morphology, physical properties, or functionality to the final supramolecular material. Finally, we wish to highlight the profound effect that the architecture of

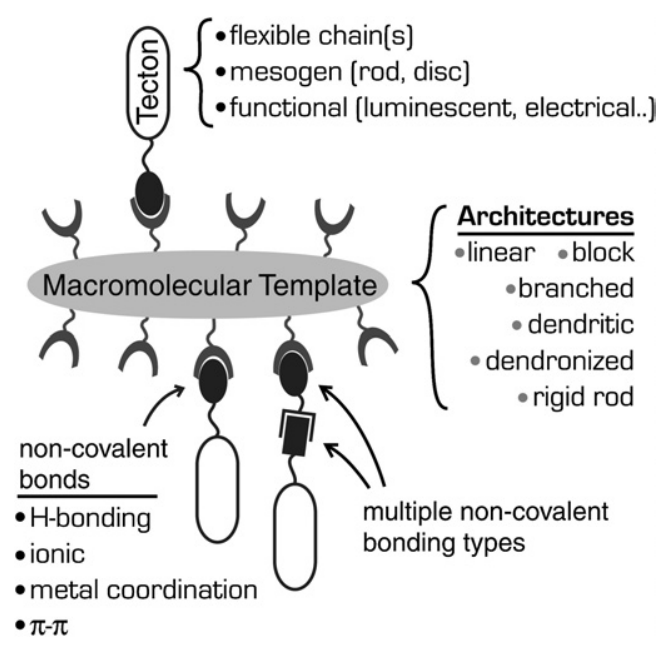

Fig. 2 Types of supramolecular polymeric complexes considered in this review.

the chosen macromolecular template can have on the resulting mesomorphic structures.

Of course, owing to space limitations, this review is not exhaustive in its subject matter. Additionally, many other supramolecular polymeric complexes which do not fall under the category of supramolecular liquid crystalline side-chain polymers have also been created. The interested reader is directed to relevant reviews of supramolecular polymeric materials created by hydrogen bonding, ${ }^{10-14}$ ionic complexation, ${ }^{15-19}$ metal coordination, ${ }^{20} \pi-\pi$ interactions, ${ }^{21}$ or combinations thereof. ${ }^{22}$

\section{Discussion}

\subsection{Hydrogen bonding}

In a seminal contribution to the field, Kato and Fréchet reported the synthesis of thermotropic nematic supramolecular polymer $\mathbf{1}$ formed via single hydrogen bonds between a polymer bearing benzoic acid side groups and a pyridine-containing mesogen. ${ }^{23}$ The resulting material showed a nematic-isotropic transition temperature significantly higher than that of either of the individual components.

However, mesomorphic materials can also be created by complexation of flexible side chains to a polymer backbone to form comb-shaped supramolecules as in e.g. 2a and $\mathbf{3}$ (Fig. 3). In 2a, 3-pentadecylphenol (PDP) is hydrogen bonded to poly(4-vinyl pyridine) (PVP), leading to a microphase separation between the the apolar surfactant alkyl tails and the more polar polymer backbone and PDP head groups. PVP(PDP $)_{x}$ complexes (where $x$ is the stoichiometric ratio of PDP molecules to PVP monomer units) showed lamellar structures with repeat distances decreasing from $c a .5 \mathrm{~nm}$ to $c a .3 .6 \mathrm{~nm}$ as $x$ was increased from 0.1 to $1.0 .^{24}$ The width of the alkyl tail domain remains constant as $x$ is varied. However, with increasing $x$, the increased grafting density forces the polymer backbone to adopt a more stretched, flat configuration, decreasing the width of the polar layers. Overall, the lamellar spacing, $D$, varied as $1 / x$. Similar results as a function of complexation degree, $x$, were obtained in experiments on complexes $\mathbf{3}$, between poly(ethylene oxide) and soft-type (branched) dodecylbenzenesulfonic acid 

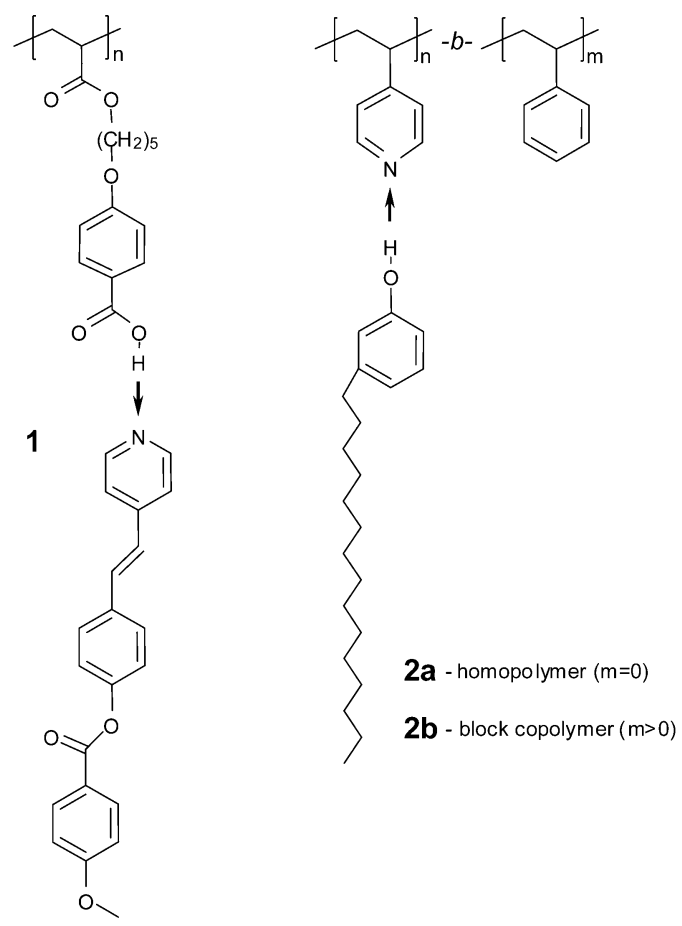

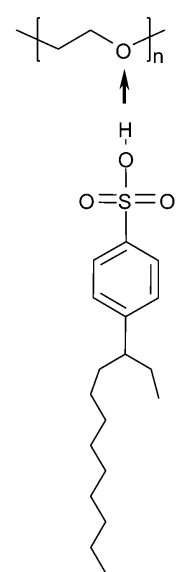

3

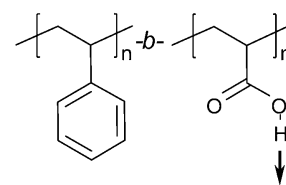<smiles>CCCCCC(C)Oc1ccc(-c2ccc(OCn3ccnc3)cc2)cc1</smiles>

Fig. 3 Examples of mesomorphic polymeric supramolecules constructed via hydrogen bonding. Hydrogen bonds are indicated by arrows pointing from H-bond donor groups to H-bond acceptor groups.

(DBSA). ${ }^{25}$ The PVP(PDP $)_{x}$ complexes undergo increasing disorder upon heating; Fig. 4 presents, for example, temperature-dependent SAXS results for PVP(PDP $)_{0.85}$, showing an order-disorder transition temperature (ODT) at $c a .65^{\circ} \mathrm{C}$, above

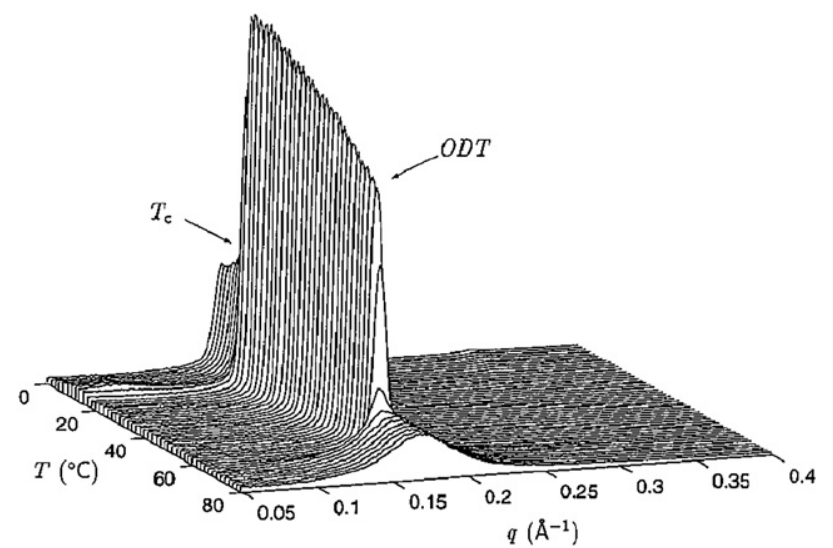

Fig. 4 Temperature dependent SAXS results for PVP(PDP $)_{0.85}$ complex (2a). Reprinted with permission from ref. 28. which a correlation hole peak ${ }^{26,27}$ owing to local concentration fluctuations is observed. ${ }^{28}$ It should be noted that this ODT is due to compatibilization of the polar and non-polar components rather than hydrogen bond breaking. The transition shown around room temperature, indicated by $T_{\mathrm{c}}$, corresponds to melting of the alkyl tails. Subsequent work with PVP and various hydrogen bond donating amphiphiles showed four regimes, outlined in Table 1, according to surfactant-polymer repulsion and hydrogen bond strength. ${ }^{29}$ Thus, the judicious choice of polymer and surfactant can be used to tune material properties.

The extension of the supramolecular comb polymer concept to include block copolymer templates is relatively straightforward (as in, e.g. 2b), and the resulting materials show hierarchically self organized structures on two length scales: that of the supramolecular comb shaped block, and that of the overall block copolymer. Fig. 5 shows a transmission electron microscopy (TEM) image of one such example, analogous to $\mathbf{2} \mathbf{b}$, but here the polystyrene- $b$-PVP template was complexed with the longer-tailed nonadecylphenol (NDP) to yield PS-PVP(NDP) $)_{1.0}$, where the PVP(NDP) comb weight fraction was $0.48 .^{30}$ The sample shows lamellar-within-lamellar morphology; that is, the PVP(NDP) combs form lamellae of period $4.5 \mathrm{~nm}$ (identical to

Table 1 Segregation regimes observed in hydrogen-bonded comblike polymers ${ }^{29}$

\begin{tabular}{llll}
\hline & Polymer backbone-surfactant repulsion & Low \\
\cline { 2 - 3 } $\begin{array}{l}\text { Hydrogen bond } \\
\text { association strength }\end{array}$ & High & $\begin{array}{l}\text { Microphase separated } \\
\text { (mesomorphous) } \\
\text { Macrophase separated }\end{array}$ & $\begin{array}{l}\text { Strongly associated isotropic mixture, } \\
\text { correlation hole peak observed in SAXS } \\
\text { Weakly associated homogeneous mixture, } \\
\text { no correlation hole peak observed in SAXS }\end{array}$ \\
\hline
\end{tabular}




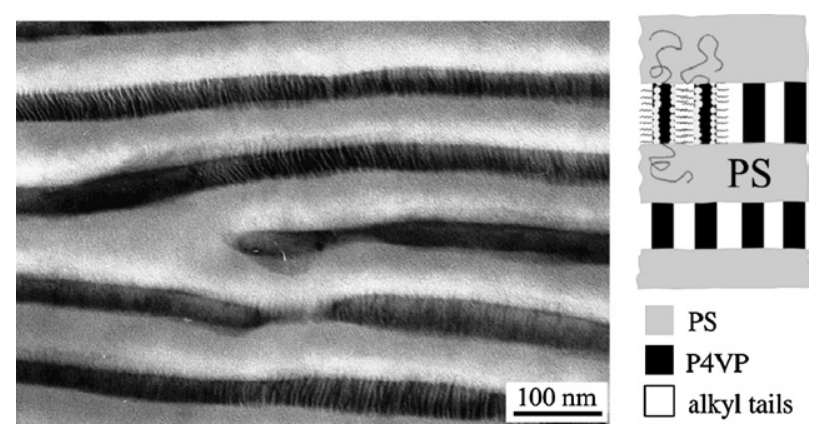

Fig. 5 TEM image showing lamellar-within-lamellar morphology in a PS-PVP(NDP) ${ }_{1.0}$ sample along with a schematic drawing detailing the hierarchical mesostructure formation. Reprinted with permission from ref. 30 .

those observed in homo-PVP(NDP) $)_{1.0}$ complexes), arranged within an overall lamellar structure of period $\sim 90 \mathrm{~nm}$. Varying the relative lengths of the PS and PVP blocks affords control over the final structure, as is typically found in block copolymer melts, and along with the lamellar-within-lamellar structure just mentioned, observed structures include lamellar-withinspherical, lamellar-within-cylindrical, lamellar-within-hexagonally perforated lamellae, gyroid-within-lamellar, cylindrical-withinlamellar, and spherical-within-lamellar. ${ }^{30-32}$ In all the above examples, however, while the long length scale morphology (i.e. at the block copolymer length scale) could be affected by varying the PS : PVP ratio, the only affordable short length scale structure (i.e. at the surfactant length scale), was lamellar. Such structure-within-structure morphologies have been observed in a few other analogous complexes based on a block copolymer template as well, including systems formed via ionic bonding, which will be discussed here later.

Other examples involving $\mathrm{H}$-bonding include cylinder-inlamellar structures observed in polybutadiene-PEO(DBSA) samples $^{33}$ (see 3), and lamellar-in-lamellar structures observed in 4, in which the poly(acrylic acid) (PAA) block of a PS- $b$ PAA diblock was functionalized with mesogenic side chains. ${ }^{34}$ This latter system showed, for fraction of occupied PAA monomer sites $x=0.8$, fast switching of domain orientation under the influence of modest AC electric fields, below the ODT, as schematically shown in Fig. 6. This domain re-orientation was not observed in PS-PAA(mesogen $)_{0.5}$ complexes, nor in a closely analogous control system where the mesogens were covalently bonded to the side-chain liquid crystal block, implying that the domain re-orientation relies on having a delicate balance between sufficient populations of both bound and free mesogens.

Indeed, the relatively low binding energy and reversibility of hydrogen bonding can be an attractive feature of this supramolecular assembly technique. Ikkala and coworkers have exploited this property to create, for example, mesoporous materials via the removal of PDP from a shear-oriented sample of a PS-PVP(PDP) (PS wt. fraction $\approx 75 \%$ ) complex showing lamellar-in-cylindrical structure. ${ }^{35}$

\subsection{Ionic bonding}

There are several methods to achieve ionic complex formation, including ion exchange in aqueous solution (typically followed

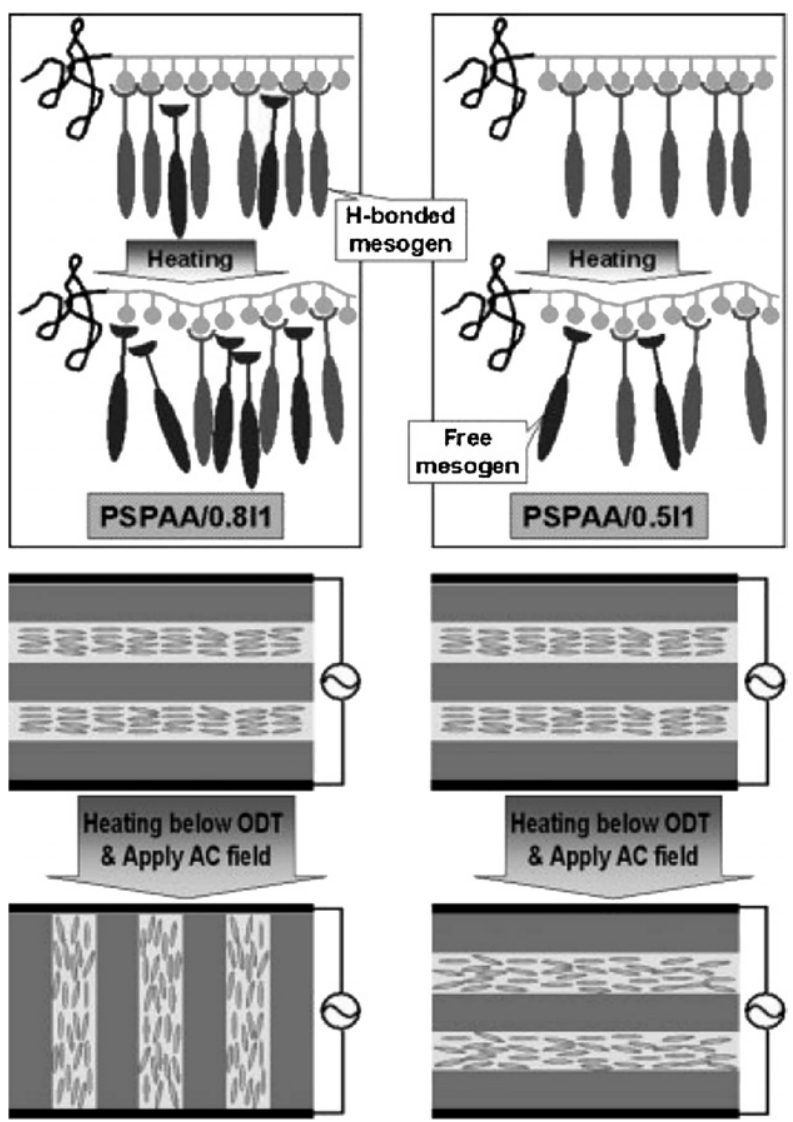

Fig. 6 Schematic showing rearrangement of PS-PAA(mesogen) $)_{x}$ hydrogen bonded complexes, $\mathbf{4}$, upon application of AC electric fields. Reprinted with permission from ref. 34 .

by precipitation), acid-base proton exchange, quaternization reactions, and redox reactions. The first of these is the most common in the literature, involving the simple mixing of oppositely charged polyelectrolyte and surfactant solutions. In what is considered the seminal publication on this subject, for example, Antonietti and coworkers reported structures of polyelectrolyte-surfactant complexes synthesized by mixing poly(styrenesulfonate) (PSS) and alkyltrimethylammonium surfactant solutions, from which $1: 1$ charge complexes precipitated $\left(\mathbf{5}+\mathbf{9}\right.$, Fig. 7). ${ }^{36}$ This method of synthesis benefits from a cooperative binding mechanism, whereby complexation of a first surfactant molecule greatly enhances the probability of another surfactant binding to a neighboring monomer site. This process reaches completion at surfactant concentrations significantly below the critical micelle concentration. ${ }^{37}$

The PSS-surfactant complexes showed lamellar arrangement with long periods varying from 2.9 to $4.1 \mathrm{~nm}$ as the surfactant tails were increased from 12 to 18 methylene units. However, indications were found which imply that the lamellar interfaces undulate, rather than being strictly planar. Such undulations are supposed to arise from a mismatch between the packing of surfactant tails and polymer backbone/ionic bonding sites, a phenomenon known as "frustration," 38 and are not uncommon for ionic complexes. In contrast, such effects are typically not observed in the hydrogen-bonded complexes discussed earlier, in which the much lower bonding strengths involved allow 

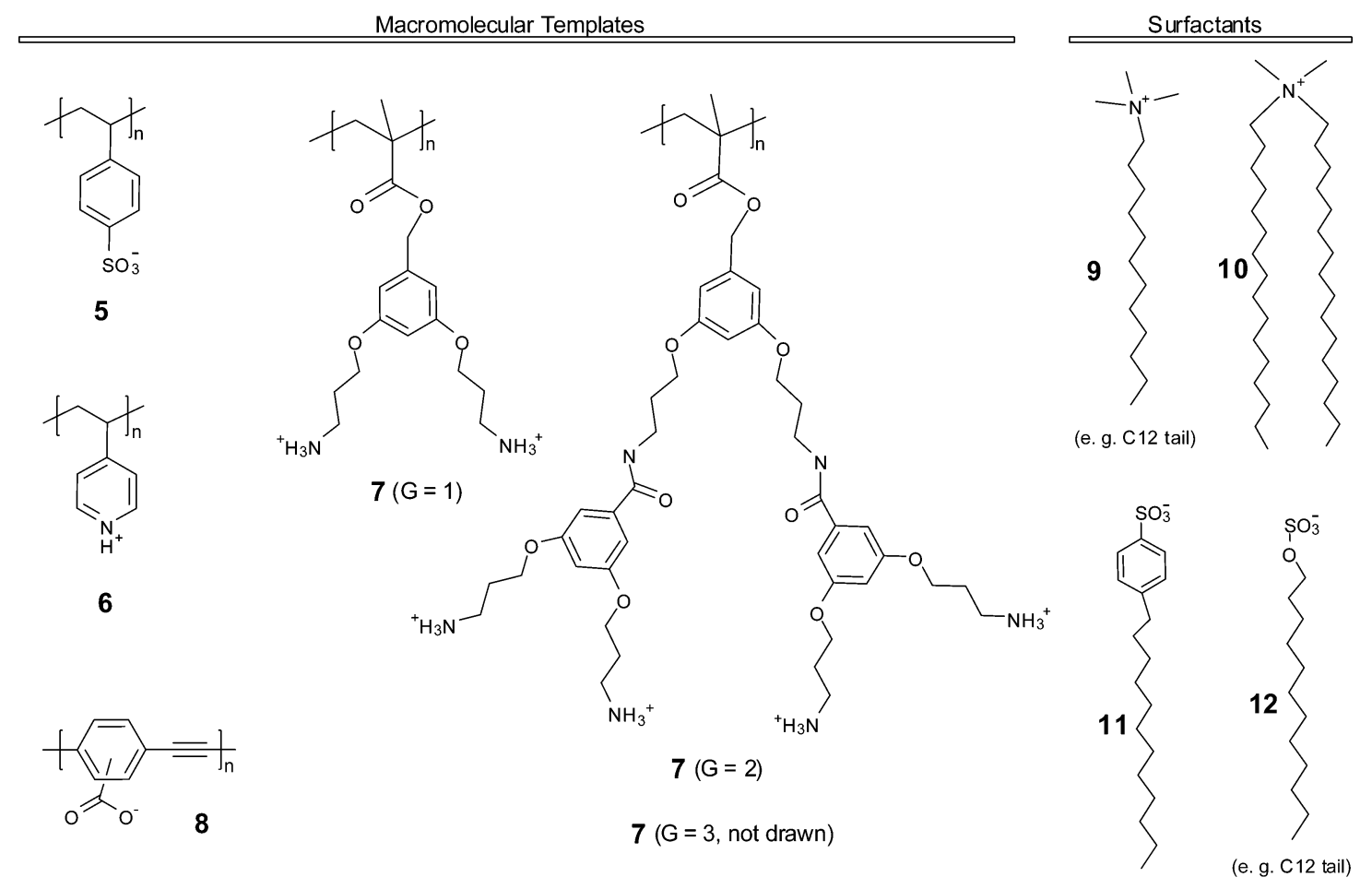

Fig. 7 Examples of some macromolecular template architectures and surfactants used in ionic complexes discussed in the text.

potential frustration effects to be mitigated by dynamic association/dissociation of surfactant.

Another dissimilarity between complexes created via hydrogen bonding and ionic bonding is observed in sub-stoichiometric complexes. Ikkala et al. prepared ionic complexes between PVP and (unbranched) para-dodecylbenzenesulfonic acid $\left[\mathrm{PVP}(\mathrm{DBSA})_{x}\right](\mathbf{6}+\mathbf{1 1}$, Fig. 7$)$ at various compositions and found lamellar mesostructures whose repeat distances increased only very weakly as $x$ was varied between 0.5 and 1.0 , in contrast to the $D \sim 1 / x$ behavior observed in the analogous hydrogenbonded $\mathrm{PVP}(\mathrm{PDP})_{x}$ complexes. ${ }^{39} \dagger$ It is suggested that the surfactants in the ionic complexes tend to form clusters, similarly to ionomer polymers, ${ }^{40}$ leading to regions along the PVP backbone of alternating high and low complexation degree, with only the former participating in the lamellar ordering. ${ }^{24}$ Such clustering of surfactants in substoichiometric complexes was shown to occur in solution (even in solvents of low polarity where ionic bonding is expected to be strongest) as well. ${ }^{41}$

Since the initial reports of such polymeric ionic complexes, extensive research efforts have greatly broadened the array of materials created by ionic self assembly, incorporating amphiphiles of increased complexity or functionality. For example, polyelectrolyte complexes with fluorinated surfactants yield mesomorphous materials with extremely low surface energies, ${ }^{19,42}$ and mesomorphous photoluminescent polyelectrolyte surfactant

$\dagger$ The PVP(DBSA $)_{x}$ complexes were formed by mixing solutions of the two components in organic solvents. A proton is transferred from the highly acidic DBSA to the basic PVP, and ionic bonding occurs between the resulting charged species. Removal of solvent yielded the solid complexes. Note that it is not possible to vary complex stoichiometry via the ion exchange method. complexes have been created using a modified diketodiphenylpyrrolopyrrole chromophore as surfactant. ${ }^{43}$ The creation of polyelectrolyte-surfactant complexes starting from polymer templates of increasingly complex structure, architecture, and functionality has also been a major focus of research efforts, and has included, among others, polypeptides, hyperbranched polymers, dendrimers, dendronized polymers, block copolymers, and rigid rod structures.

Poly(L-glutamate)/alkyltrimethylammonium $(x=1.0$, alkyl tail lengths of $\mathrm{C} 12, \mathrm{C} 16$, and $\mathrm{C} 18$ ) complexes showed a lamellar arrangement of alternating layers of $\alpha$-helical polypeptide and perpendicular, interdigitated, and fully extended alkyl chains. ${ }^{44,45}$ Crystallization in the alkyl tails was found in the $\mathrm{C} 18$ case only. Analogous complexes of Poly(L-lysine)/dodecylsulfate $(x=1.0)$ also showed lamellar organization, however the polypeptide backbones adopted a mixture of $\alpha$-helix and $\beta$-sheet conformations, with the $\alpha$ vs. $\beta$ content being highly dependent on the conditions of the solvent from which the solid samples were re-cast. ${ }^{46}$ Ionic complexes of polylysines and alkyl sulfate surfactants, in which the secondary structure of the polypeptide is virtually completely suppressed, have been recently reported by Mezzenga et al. using hyperbranched polylysines as macromolecular polyelectrolytes. ${ }^{47}$ Depending on the length of the alkyl tail used, these systems showed, at length scales similar to alkyl tail contour lengths, both lamellar packing (C18), and a more unconventional columnar hexagonal packing (C12). Results were interpreted by the intrinsic curvature induced at the hyperbranched polylysine-alkyl tail complex, which differs markedly compared to analogue systems obtained by linear polyelectrolytes.

Systems in which the degree of branching of the macromolecular polyelectrolyte is systematically controlled have also been 
investigated by a number of authors. For example, Serrano et al. have investigated two families of dendrimer templates, poly(amidoamine) (PAMAM) and poly(propylene imine) (PPI), each bearing terminal amine groups, when complexed with $\mathrm{C} 18$ alkanoic acid surfactants via the acid-base route, while also varying the dendrimer generation, $G{ }^{48}$ Complexes of PPI with $\mathrm{C} 14$ and $\mathrm{C} 10$ alkanoic acids were also reported in the same study. In all but two cases, these complexes showed smectic A phases. For the PAMAM(C18) complexes, the repeat distance increased with dendrimer generation. For the $\operatorname{PPI}(\mathrm{Cn})$ complexes, however, the repeat distance was found to be essentially independent of $G$, while increasing as expected with increasing surfactant length. The PPI(C18) complexes of highest generation (32 and 64 surfactants per dendrimer) showed columnar phases-hexagonal and tetragonal, respectively. All these mesomorphous structures persisted until at least $100{ }^{\circ} \mathrm{C}$, although a certain amount of (covalent) amide bond formation (via condensation and of the ammonium and alkanoate species) occurred at high temperature, resulting in a lowering of the clearing temperature.

Complexes between cationic dendronized polymers of generation 1-3 (compounds 7, Fig. 7) and anionic alkyl surfactants (C8 to $\mathrm{C} 18$ lengths, e.g. 12, Fig. 7) have also been reported recently, showing a panoply of mesostructures depending on template generation and surfactant length. ${ }^{49,50}$ These are diagrammed in Fig. 8, and notably include unusual columnar rectangular and tetragonal phases. The clearing temperatures of the various structures were found to be highly dependent on the topology of the liquid crystalline phase investigated, as shown in Fig. 9. Real space TEM images of the columnar structures showed the surprising result that despite being the majority component in terms of volume fraction, the domains composed of surfactant alkyl tails formed the dispersed phase, i.e. the column cores. ${ }^{51}$ Consequently, it has been argued that, upon removal of the ionically bound alkyl tails, these systems can be used as ideal systems to design ultra-dense porous materials with controllable patterns and lattices. ${ }^{52}$

Block copolymer templates with one neutral and one polyelectrolyte block have also been explored for ionic complexation,

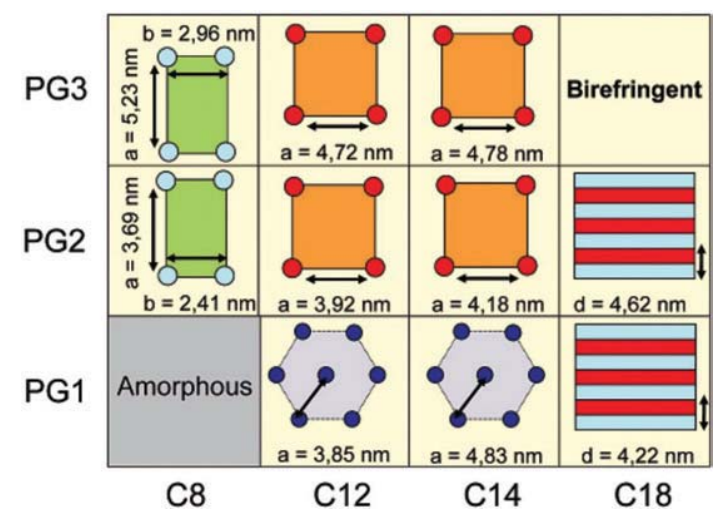

Fig. 8 State diagram describing the mesomorphic structures observed in dendronized polymer-surfactant complexes with varying dendron generation (vertical axis) and surfactant tail length (orizontal axis). The symmetry and lattice parameters are indicated. Reprinted with permission from ref. 50 .

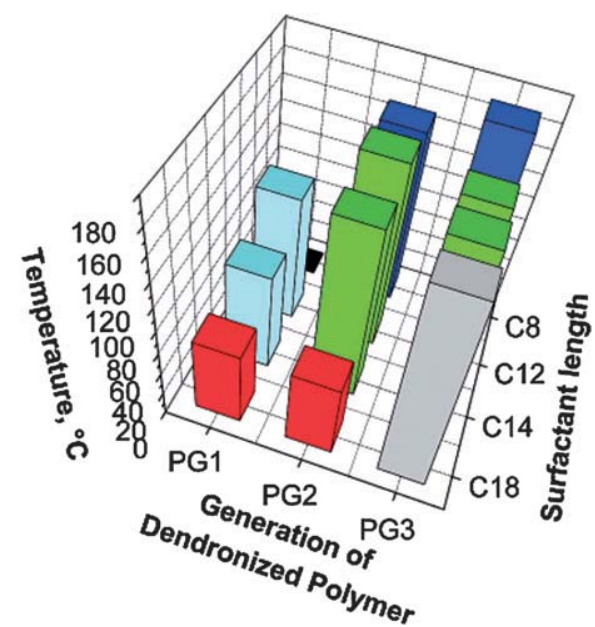

Fig. 9 Chart of liquid crystalline-isotropic transition temperatures observed in dendronized polymer-surfactant complexes as determined by SAXS experiments. Reprinted with permission from ref. 50

resulting in hierarchically ordered structures analogous to the previously discussed hydrogen bonded BC-amphiphile complexes. For example, complexes of poly(ethylene oxide)- $b$ poly(ethylene imine)-dodecanoate were shown to form lamellar-within-lamellar structures. ${ }^{53}$ While the parameter space of block copolymer volume fraction and molecular weight has not been explored as thoroughly in ionically complexed systems as in the PS-PVP(alkylphenol) (H-bonded) system, there remain several other reports that are worth noting in this area. For instance, the employment of polypeptide-based block copolymers as templates has yielded materials incorporating polypeptide secondary structural motifs into hierarchically (structure-within-structure) ordered morphologies. ${ }^{54,55}$ Elsewhere, Chen et al. recently reported hierarchical cylinder(hexagonal)within-lamellar ordering in ionically bonded PS-PVP(DBSA $)_{1.0}$ complexes, and much more unconventional tetragonallypacked-cylinder-within-lamellar ordering for PS-PVP(DBSA $)_{0.5-0.6}$ complexes. ${ }^{56}$ It was further shown that, in the substoichiometric case, the PVP(DBSA) (low-length scale) lamellae were oriented in the same plane as the cylinder axes, as opposed to the typical arrangement where the supramolecular comb lamellae are oriented perpendicular to the cylinder axes. It was claimed that this unusual structure is made possible by the ability of the ionically bound surfactants, in the substoichiometric case, to segregate into distinct domains, as previously discussed. Thus the PS-PVP(DBSA) $)_{0.5-0.6}$ complexes eventually adopt a triblock-like composition, i.e. PS- $b$-PVP- $b$-PVP(DBSA $)_{x} \approx 1.0$. Given the rich variety of complex mesophases predicted ${ }^{57}$ for "simple" A-B-C triblock copolymers, the possibilities to design long-length scale topologies-within-lamellar here are truly vast.

Conjugated polymers having rigid backbones have also served as macromolecular templates for ionic complexation. The blue-emitting electroluminescent polymer poly(1,4-phenyleneethinylenecarboxylate) (8, Fig. 7), itself a brittle material as a solid, formed easily processable, flexible complexes when complexed with dihexadecyldimethylammonium surfactants (10, Fig. 7), showing lamellar microstructure. ${ }^{58}$ 


\subsection{Multiple bonding schemes: ionic and hydrogen bonding}

By now it is well known that attachment of alkyl chains to conjugated polymer backbones greatly improves the solubility, processability, and mechancial properties of this important class of materials. As in the previous example, supramolecular chemistry offers an enticing route to achieve this. However, due to the very strong tendency of mixtures of rigid rods and random coils to macrophase separate, ${ }^{59}$ supramolecular attachment of side-chain coils to rigid rod polymers requires a strong association energy, ${ }^{60}$ achievable with ionic bonding, but not via single hydrogen bonds. Nevertheless, several beautiful examples exist where supramolecular ionic functionalization of a rigid rod was followed by multiple hydrogen bonding of a suitable surfactant, resulting in homogeneous, mesostructured materials. ${ }^{61,62}$ In one example, Ikkala et al. reported hexagonal organization of complexes of polyaniline (PANI), first complexed ionically with camphorsulfonic acid (CSA) and then complexed with the multiple hydrogen bond donating 4-hexylresorcinol (Hres) (see 13, Fig. 10). ${ }^{61}$ In this case, the ionic complexation of the CSA provides the dual benefit of enabling $\mathrm{H}$-bonding acceptor sites for the Hres and doping the PANI, which is necessary to obtain a conducting material.

Another very notable body of work on combining ionic and hydrogen bonding to generate supramolecular polymers involves PVP ionically complexed with methanesulfonic acid (MSA) and subsequently complexed to alkylphenols such as PDP via hydrogen bonding and related block copolymers starting from PS-PVP templates (14, Fig. 10). PVP(MSA $)_{1.0}(\mathrm{PDP})_{x}$ complexes show complicated phase behavior. Starting from the ordered lamellar phase at low temperatures, the system undergoes an ODT around $100-120{ }^{\circ} \mathrm{C}$. At $170{ }^{\circ} \mathrm{C}$, the PDP macrophase separates from the PVP(MSA) $)_{1.0}$ complex, becomes miscible

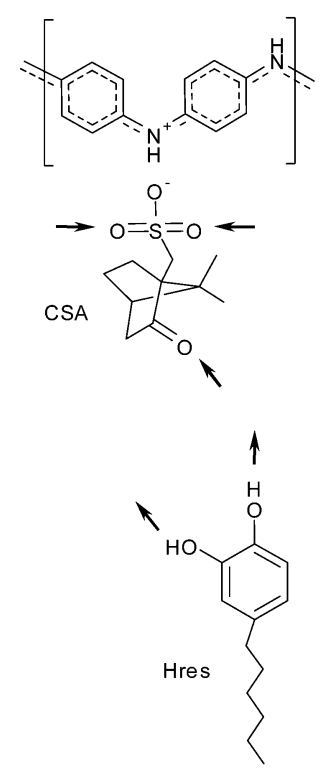

13
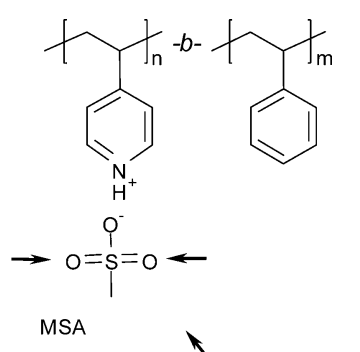

14

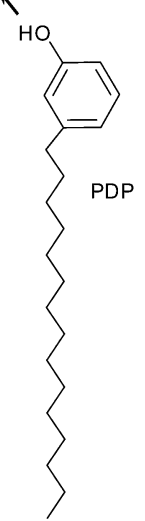

Fig. 10 Examples of complexes incorporating ionic bonding and hydrogen bonding. Dark arrows point away from hydrogen bond donors and towards hydrogen bond acceptors. again above $195^{\circ} \mathrm{C}$, and at still higher temperatures macrophase separates once again. ${ }^{63}$ Since PS and PDP are fully miscible above $130{ }^{\circ} \mathrm{C}$, the hierarchically ordered block copolymer-based complexes, PS-PVP(MSA $)_{1.0}(\mathrm{PDP})_{x}$, in addition to showing the ODT related to the comb block at $100{ }^{\circ} \mathrm{C}$, show order-order transitions at higher temperatures as significant amounts of unbound PDP begin to swell the PS domains. This complicated phase behavior has been exploited to create materials with temperature-sensitive protonic conductivity, ${ }^{63}$ and temperaturesensitive photonic bandgap. ${ }^{64}$ In the latter case, for example, a PS-PVP(MSA $)_{1.0}(\mathrm{PDP})_{1.5}$ complex was prepared which showed lamellar-in-lamellar morphology with a long period of ca. $160 \mathrm{~nm}$, resulting in a photonic bandgap in the green region of the spectrum. Upon heating above $125^{\circ} \mathrm{C}$, the morphology changed to simple lamellar, in which both the PS and PVP(MSA) $)_{1.0}$ phases were swollen with PDP. The long period was reduced to $c a$. $117 \mathrm{~nm}$, shifting the bandgap to much lower wavelength, out of the visible range.

\subsection{Multiple bonding schemes: ionic and $\pi-\pi$ bonding}

The tendency of $\pi-\pi$ bonding to drive liquid crystallinity in discor rod-shaped polyaromatic molecules is by now well known. ${ }^{21,65}$ Along these lines, for example, Sary et al. have exploited $\pi-\pi$ interactions in $\pi$-conjugated rod-coil block copolymers to drive the formation of lamellar clusters within a continuous phase of rod-coil block copolymer stable in an isotropic homogeneous phase. ${ }^{66}$ In fact, due to the large polarizability and good intermolecular contact owing to the rigid and anisotropic shape of such molecules, dispersive forces play a large role in liquid crystalline structure formation in such systems, as well. Since the effects of arene-arene bonding and these dispersive forces always act in tandem, we adopt for simplicity the term " $\pi-\pi$ bonding" as a shorthand to describe the combined effects, even though it is incorrect, strictly speaking. However, although sufficient bond strengths are possible, ${ }^{67} \pi-\pi$ bonding lacks the specificity necessary to create the sort of well-defined side-chain bonded LCP's discussed here. However, several interesting examples exist of complexes where polyaromatic side-chains are grafted to a main polymer chain via ionic bonding and are driven by $\pi-\pi$ interactions to adopt even more highly ordered configurations. In a series of papers, Thünemann et al. complexed a carboxylic acid-modified peri-hexabenzocoronene (HBC, 15, Fig. 11), to various polycation backbones. ${ }^{68-70}$ Variously substituted HBC's by themselves are in general widely
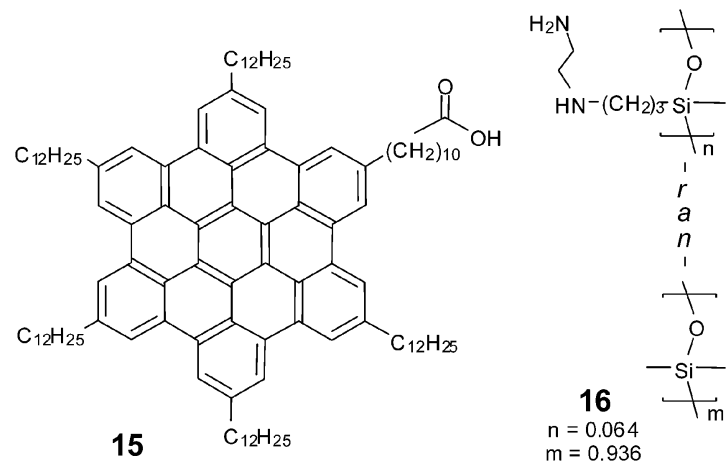

Fig. 11 Example of a complex including ionic and $\pi-\pi$ bonding. 


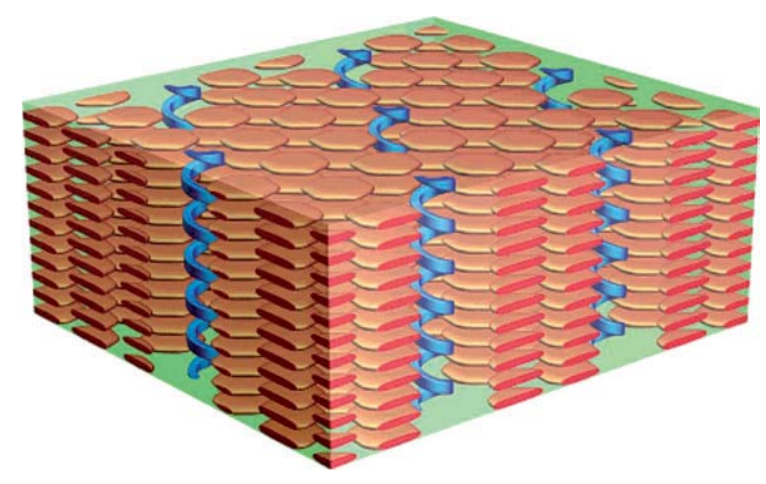

Fig. 12 Schematic drawing of superstructure formation in PEO- $b$ PLL(15) $)_{1.0}$ complex. PLL $\alpha$-helices are drawn in blue, while modified HCB's (15) are drawn as red-orange discs. Reprinted with permission from ref. 70

studied, as they form columnar liquid crystalline phases exhibiting high values of intrinsic charge carrier mobility. ${ }^{\mathbf{7 1}}$ Complexation of the commercially available partially aminoethylaminopropyl-functionalized polysiloxane, 16, with $\mathbf{1 5}$ resulted in columnar phases which showed higher intracolumnar order than the uncomplexed HCB, 15. ${ }^{69}$ Note the extremely low fraction of available backbone sites for ionic bonding in 16, 0.064; complexation of this fraction of alkyl tail-bearing surfactants will not induce mesomorphism, ${ }^{24}$ highlighting the profound effects of the $\pi-\pi$ interactions in this case. Complexation of $\mathbf{1 5}$ with a PEO-poly(L-lysine) (PEO-PLL) block copolymer formed even more highly ordered structures. The PLL backbones, which adopted $\alpha$-helical secondary structures, were shown to be surrounded symmetrically by 6 HCB columns, and these hexagonal units packed in a larger hexagonal superstructure, as schematized in Fig. 12. ${ }^{70}$

\subsection{Metal coordination}

Metal coordination has been exploited extensively in supramolecular chemistry. ${ }^{20,72-74}$ Efforts at creating the types of materials considered in this work via metal coordination, although much more rare up to this point, are beginning to accelerate. Ikkala et al. reported complexes between PVP and zinc dodecylbenzenesulfonate, $\operatorname{PVP}\left(\mathrm{Zn}(\mathrm{DBS})_{2}\right)_{x}$, which showed lamellar structures much like the PVP(DBSA) ionic complexes previously discussed. ${ }^{75}$ Removal of the $\mathrm{Zn}(\mathrm{DBS})_{2}$ from PS-PVP $\left(\mathrm{Zn}(\mathrm{DBS})_{2}\right)$ complexes by selective solvent washing resulted in stable mesoporous materials. ${ }^{76}$ The advantage of using the metal coordination rather than the simple H-bonding discussed earlier was that the higher bond strength allows bonding of larger units, leading to materials with larger porosity.

Another macromolecular template with the potential to create metal-coordinated liquid crystalline materials is poly(norbornene) functionalized with palladated sulfur-carbon-sulfur pincers, 17 (Fig. 13), synthesized by Weck and Pollino. ${ }^{77}$ This template was complexed with mesogens bearing nitrile or pyridine groups, although no report of resulting mesophases was made. It was noted that, due to the stronger binding of the pyridine-metal complex relative to the nitrile-Pd complex, the pyridine-based mesogens could be made to displace the

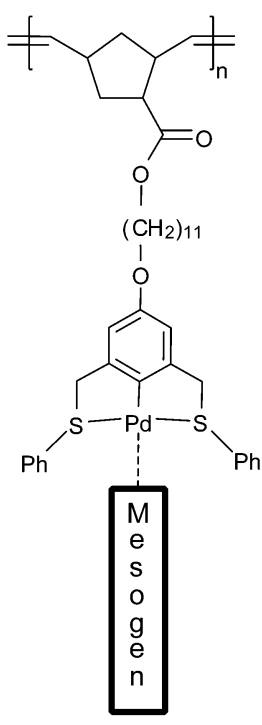

17

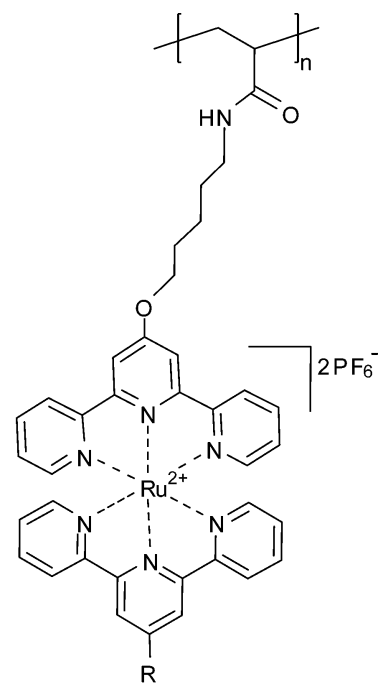

$18 \mathbf{a} \mathrm{R}=-\mathrm{O}\left(\mathrm{CH}_{2}\right){ }_{15} \mathrm{CH}_{3}$ $18 b \mathrm{~b}=\mathrm{H}$
Fig. 13 Examples of side-chain supramolecular polymers created via metal coordination.

nitrile-based mesogens, raising the possibility of responsive materials.

Polymers recently synthesized in the groups of Tew ${ }^{78-81}$ and Kallitsis ${ }^{82}$ bearing pendant terpyridine (terpy) groups are perhaps even more exceptional candidates; terpy-metal complexes impart many interesting luminescent, thermochromic, or magnetic properties, depending on the choice of metal. Metal-to-ligand charge transfer (MLCT) bands are observed in transition metal-terpy complexes, while lanthanide-terpy complexes show ligand-to-metal charge transfer bands (LMCT) ${ }^{83}$ Metal-coordinated polymers are therefore interesting candidates for designing materials where photo-induced charge injection or photoinduced current are required. Photochromic properties of these materials are also remarkable. In one case, such terpy side chain-bearing polymers showed excellent pink emission when complexed with $\mathrm{Eu}^{3+}$, green when complexed with $\mathrm{Tb}^{3+}$, and unique yellow emission when complexed with a mixture of both lanthanides simultaneously. ${ }^{79}$ In the first report of mesomorphism in this type of supramolecular complex, 18a (Fig. 13) was birefringent and was shown by SAXS to self-assemble into a hexagonal phase with repeat spacing $5.1 \mathrm{~nm}$, whereas complex $\mathbf{1 8 b}$ showed no mesomorphism. ${ }^{\mathbf{1}}$

\subsection{Multiple bonding strategies incorporating metal coordination}

As metal-ligand complexes are often charged, the door is open to the addition of additional complexation via ionic bonding. Ikkala et al. showed that mixtures of PVP, $\mathrm{Zn}(\mathrm{DBS})_{2}$, and 2,6bis(octylaminomethyl)pyridine (BOAP) formed stable complex 19 (Fig. 14). ${ }^{84}$ SAXS experiments on 19 showed well-ordered cylindrical structures (4 higher-order scattering peaks were observed) with a $d$-spacing of $2.1 \mathrm{~nm}$. The transition from lamellar structures observed in $\operatorname{PVP}\left(\mathrm{Zn}(\mathrm{DBS})_{2}\right)_{1.0}$ to the hexagonal structure adopted by $\mathbf{1 9}$ is presumably due to increased 


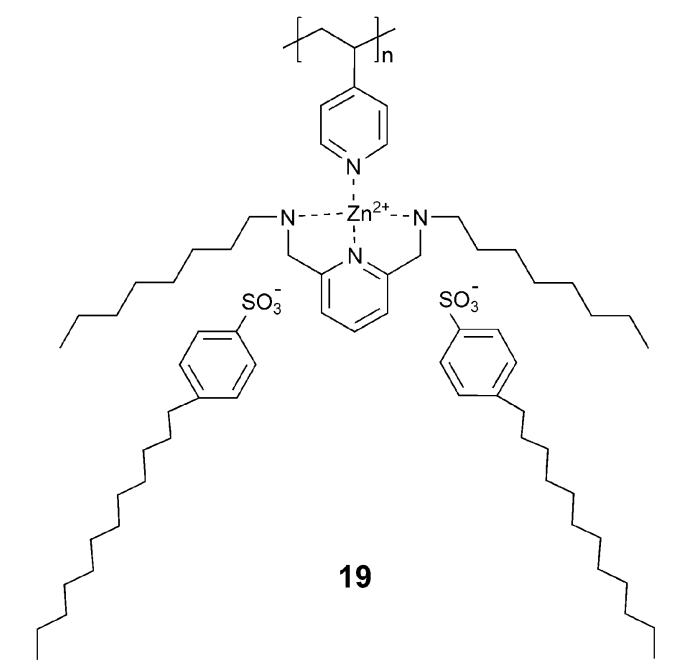

Fig. 14 Structure of $\mathrm{PVP}\left(\mathrm{Zn}(\mathrm{BOAP}) \mathrm{DBS}_{2}\right)$ complex reported by Ikkala et al. ${ }^{84}$

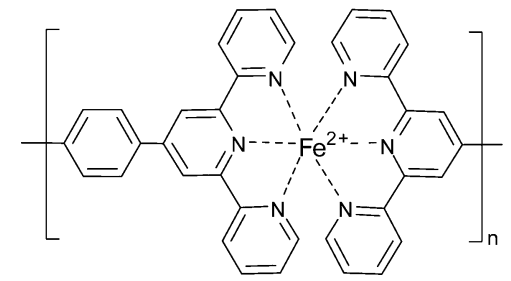

20

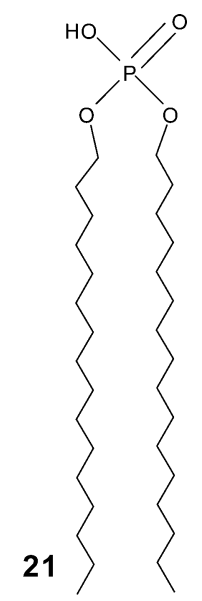

Fig. 15 Components of metallo-supramolecular polymer-surfactant complexes reported by Kurth et al. ${ }^{85}$

crowding around the polymer backbone due to the extra alkyl tails.

Another interesting example comes from the group of Kurth, who synthesized the supramolecular coordination polymer $\mathbf{2 0}$, and complexed it to the surfactant dihexadecylphosphate (DHP, 21, Fig. 15) ${ }^{85}$ Interestingly, the complexes showed $\sim 6$ DHP molecules per polymer repeat unit, rather than the minimum of two needed for charge neutralization. The other surfactants must be kept in place via hydrogen bonding (note that DHP contains both H-bond donor and acceptor sites). Solid state structures of these complexes showed lamellar ordering with a repeat distance of $3.3 \mathrm{~nm}$, implying full interdigitation of DHP alkyl chains, which show a certain amount of crystallinity in their packing. ${ }^{86}$ When prepared as multilayer films by the Langmuir-Blodgett technique, the complex shows a reversible phase transition near $55^{\circ} \mathrm{C}$, corresponding to melting of the alkyl tails. ${ }^{87}$ This transition induces a slight increase in the average $\mathrm{Fe}-\mathrm{N}$ bond distance, which results in a diamagneticparamagnetic transition (from a low-spin to a high-spin state) ${ }^{88}$

\section{Summary and outlook}

Supramolecular chemistry offers a wide variety of tools to create self-assembled materials with complex topologies. In this review we have provided a sampling of the types of structures that have been demonstrated in side-chain type liquid crystalline polymers "synthesized" by complexing low molecular weight species to a macromolecular template via the various non-covalent interactions that give supramolecular chemistry its great potential. In doing so, we hope to have demonstrated how the various parameters - type of non-covalent bonding, macromolecular template architecture, and surfactant/side-chain propertiescan be judiciously chosen to affect the structures, topologies and properties of the final materials designed. We have also attempted to highlight efforts which take advantage of multiple bonding strategies, synergistically combining the strengths of each respective technique. By fully exploiting the combination of all possible macromolecular architectures, with the available structures of low molecular weight compounds and the specific supramolecular routes (or combination thereof) used to bind these together, one has potentially access to an endless panel of possibilities to design complex structures and functionalities, where the only limit is likely to be our own imagination.

\section{Acknowledgements}

Financial support from the Swiss National Science Foundation is gratefully acknowledged.

\section{References}

1 J. M. Lehn, Angew. Chem., Int. Ed. Engl., 1988, 27, 89.

2 G. M. Whitesides, J. P. Mathias and C. T. Seto, Science, 1991, 254, 1312.

3 M. Muthukumar, C. K. Ober and E. L. Thomas, Science, 1997, 277, 1225.

4 I. W. Hamley, The Physics of Block Copolymers, Oxford University Press, Oxford, UK, 1998.

5 K. Inomata, M. Haraguchi and T. Nose, Polymer, 1996, 37, 4223.

6 B. G. G. Lohmeijer and U. S. Schubert, Angew. Chem., Int. Ed., 2002, 41, 3825 .

7 W. H. Binder, S. Bernstorff, C. Kluger, L. Petraru and M. J. Kunz, Adv. Mater., 2005, 17, 2824.

8 O. Ikkala and G. ten Brinke, Chem. Commun., 2004, 2131.

9 L. Brunsveld, B. J. B. Folmer, E. W. Meijer and R. P. Sijbesma, Chem. Rev., 2001, 101, 4071.

10 W. H. Binder and R. Zirbs, Adv. Polym. Sci., 2007, 207, 1.

11 L. Bouteiller, Adv. Polym. Sci., 2007, 207, 79.

12 G. ten Brinke, J. Ruokolainen and O. Ikkala, Adv. Polym. Sci., 2007, 207, 113.

13 G. Armstrong and M. Buggy, J. Mater. Sci., 2005, 40, 547.

14 H. Xu, S. Srivastava and V. A. Rotello, Adv. Polym. Sci., 2007, 207, 179.

15 C. K. Ober and G. Wegner, Adv. Mater., 1997, 9, 17.

16 W. J. MacKnight, E. A. Ponomarenko and D. A. Tirrell, Acc. Chem. Res., 1998, 31, 781.

17 J. Kötz, S. Kosmella and T. Beitz, Prog. Polym. Sci, 2001, 26, 1199.

18 C. F. J. Faul and M. Antonietti, Adv. Mater., 2003, 15, 673.

19 A. F. Thünemann, Prog. Polym. Sci., 2002, 27, 1473.

20 U. S. Schubert and C. Eschbaumer, Angew. Chem., Int. Ed., 2002, 41, 2892.

$21 \mathrm{~F}$. J. M. Hoeben, P. Jonkheijm, E. W. Meijer and A. P. H. J. Schenning, Chem. Rev., 2005, 105, 1491.

22 J. M. Pollino and M. Weck, Chem. Soc. Rev., 2005, 34, 193.

23 T. Kato and J. M. J. Fréchet, Macromolecules, 1989, 22, 3818.

24 J. Ruokolainen, G. ten Brinke, O. Ikkala, M. Torkkeli and R. Serimaa, Macromolecules, 1996, 29, 3409. 
25 H.-L. Chen, C.-C. Ko and T.-L. Lin, Langmuir, 2002, 18, 5619.

26 P.-G. De Gennes, Scaling Concepts in Polymer Physics, Cornell University Press, Ithaca, 1979.

27 L. Leibler, Macromolecules, 1980, 13, 1602.

28 J. Ruokolainen, M. Torkkeli, R. Serimaa, B. E. Komanschek, O. Ikkala and G. ten Brinke, Phys. Rev. E, 1996, 54, 6646.

29 J. Ruokolainen, M. Torkkeli, R. Serimaa, S. Vahvaselkä, M. Saariaho, G. ten Brinke and O. Ikkala, Macromolecules, 1996, 29, 6621.

30 J. Ruokolainen, G. ten Brinke and O. Ikkala, Adv. Mater., 1999, 11, 777.

31 J. Ruokolainen, M. Saariaho, O. Ikkala, G. ten Brinke, E. L. Thomas, M. Torkkeli and R. Serimaa, Macromolecules, 1999, 32, 1152.

32 S. Valkama, T. Ruotsalainen, A. Nykanen, A. Laiho, H. Kosonen, G. tenBrinke, O. Ikkala and J. Ruokolainen, Macromolecules, 2006 , 39, 9327.

33 C.-S. Tsao and H.-L. Chen, Macromolecules, 2004, 37, 8984.

34 C.-Y. Chao, X. Li, C. K. Ober, C. Osuji and E. L. Thomas, Adv Funct. Mater., 2004, 14, 364.

35 R. Mäki-Ontto, K. de Moel, W. de Odorico, J. Ruokolainen, M. Stamm, G. ten Brinke and O. Ikkala, Adv. Mater., 2001, 13, 117

36 M. Antonietti, J. Conrad and A. Thünemann, Macromolecules, 1994, 27, 6007.

37 K. Hayakawa, J. P. Santerre and J. C. T. Kwak, Macromolecules, $1983, \mathbf{1 6}, 1642$.

38 P.-G. De Gennes, J. Phys. Lett., 1983, 44, L657.

39 O. Ikkala, J. Ruokolainen, G. ten Brinke, M. Torkkeli and R. Serimaa, Macromolecules, 1995, 28, 7088.

40 A. Eisenberg and J.-S. Kim, Introduction to Ionomers, WileyInterscience, New York, 1998.

41 K. N. Bakeev, Y. M. Shu, A. B. Zezin, V. A. Kabanov, A. V. Lezov, A. B. Mel'nikov, I. P. Kolomiets, E. I. Rjumtsev and W. J. MacKnight, Macromolecules, 1996, 29, 1320.

42 M. Antonietti, S. Henke and A. Thünemann, Adv. Mater., 1996, 8, 41.

43 M. Behnke and B. Tieke, Langmuir, 2002, 18, 3815.

44 E. A. Ponomarenko, A. J. Waddon, K. N. Bakeev, D. A. Tirrell and W. J. MacKnight, Macromolecules, 1996, 29, 4340.

45 E. A. Ponomarenko, A. J. Waddon, D. A. Tirrell and W. J. MacKnight, Langmuir, 1996, 12, 2169.

46 E. A. Ponomarenko, D. A. Tirrell and W. J. MacKnight, Macromolecules, 1996, 29, 8751.

47 N. Canilho, M. Scholl, H.-A. Klok and R. Mezzenga, Macromolecules, 2007, 40, 8374.

48 R. Martin-Rapun, M. Marcos, A. Omenat, J. Barbera, P. Romero and J. L. Serrano, J. Am. Chem. Soc., 2005, 127, 7397.

49 N. Canilho, E. Kasëmi, R. Mezzenga and A. D. Schlüter, J. Am Chem. Soc., 2006, 128, 13998.

50 N. Canilho, E. Kasëmi, A. D. Schlüter and R. Mezzenga, Macromolecules, 2007, 40, 2822.

51 N. Canilho, E. Kasëmi, A. D. Schlüter, J. Ruokolainen and R. Mezzenga, Macromolecules, 2007, 40, 7609.

52 N. Canilho, E. Kasëmi, A. D. Schlüter, J. Ruokolainen and R. Mezzenga, Macromol. Symp., in press.

53 A. F. Thünemann and S. General, Macromolecules, 2001, 34, 6978.

54 S. Hanski, N. Houbenov, J. Ruokolainen, D. Chondronicola, H. Iatrou, N. Hadjichristidis and O. Ikkala, Biomacromolecules, 2006, 7, 3379.

55 M. R. Hammond, H.-A. Klok and R. Mezzenga, Macromol. Rapid Commun., 2008, 29, 299.
56 H.-L. Chen, J.-S. Lu, C.-H. Yu, C.-L. Yeh, U.-S. Jeng and W.-C. Chen, Macromolecules, 2007, 40, 3271.

57 F. S. Bates and G. H. Fredrickson, Phys. Today, 1999, 52, 32.

58 A. F. Thünemann, Adv. Mater., 1999, 11, 127.

59 P. J. Flory, Macromolecules, 1978, 11, 1138.

60 A. Subbotin, R. Stepanyan, M. Knaapila, O. Ikkala and G. ten Brinke, Eur. Phys. J. E, 2003, 12, 333.

61 H. Kosonen, J. Ruokolainen, M. Knaapila, M. Torkkeli, K. Jokela, R. Serimaa, G. ten Brinke, W. Bras, A. P. Monkman and O. Ikkala, Macromolecules, 2000, 33, 8671.

62 O. Ikkala, M. Knaapila, J. Ruokolainen, M. Torkkeli, R. Serimaa, K. Jokela, L. Horsburgh, A. Monkman and G. ten Brinke, Adv. Mater., 1999, 11, 1206.

63 J. Ruokolainen, R. Mäkinen, M. Torkkeli, T. Mäkelä, R. Serimaa, G. ten Brinke and O. Ikkala, Science, 1998, 280, 557.

64 S. Valkama, H. Kosonen, J. Ruokolainen, T. Haatainen, M. Torkkeli, R. Serimaa, G. ten Brinke and O. Ikkala, Nat. Mater., 2004, 3, 872.

65 S. Chandrasekhar and G. S. Ranganath, Adv. Phys., 1986, 35, 507.

66 N. Sary, R. Mezzenga, C. Brochon, G. Hadziioannou and J. Ruokolainen, Macromolecules, 2007, 40, 3277.

67 O. A. von Lilienfeld and D. Andrienko, J. Chem. Phys., 2006, 124, 054307.

68 A. F. Thünemann, D. Ruppelt, S. Ito and K. Müllen, J. Mater. Chem., 1999, 9, 1055

69 A. F. Thünemann, D. Ruppelt, C. Burger and K. Müllen, J. Mater. Chem., 2000, 10, 1325.

70 A. F. Thünemann, S. Kubowicz, C. Burger, M. D. Watson, N. Tchebotareva and K. Müllen, J. Am. Chem. Soc., 2003, 125, 352.

71 A. M. van de Craats and J. M. Warman, Adv. Mater., 2001, 13, 130.

72 B. J. Holliday and C. A. Mirkin, Angew. Chem., Int. Ed., 2001, 40, 2022.

73 S. Leininger, B. Olenyuk and P. J. Stang, Chem. Rev., 2000, 100, 853.

74 B. G. G. Lohmeijer and U. S. Schubert, J. Polym. Sci., Part A: Polym. Chem., 2003, 41, 1413.

75 J. Ruokolainen, J. Tanner, G. ten Brinke, O. Ikkala, M. Torkkeli and R. Serimaa, Macromolecules, 1995, 28, 7779.

76 S. Valkama, T. Ruotsalainen, H. Kosonen, J. Ruokolainen, M. Torkkeli, R. Serimaa, G. ten Brinke and O. Ikkala, Macromolecules, 2003, 36, 3986.

77 J. M. Pollino and M. Weck, Synthesis, 2002, 1277.

78 K. A. Aamer and G. N. Tew, Macromolecules, 2004, 37, 1990.

79 R. Shunmugam and G. N. Tew, J. Am. Chem. Soc., 2005, 127, 13567.

80 K. A. Aamer and G. N. Tew, J. Polym. Sci., Part A: Polym. Chem., 2007, 45, 1109.

81 K. A. Aamer and G. N. Tew, Macromolecules, 2007, 40, 2737.

82 N. P. Tzanetos, A. K. Andreopoulou and J. K. Kallitsis, J. Polym. Sci., Part A: Polym. Chem., 2005, 43, 4838.

83 V. Balzani, A. Juris, M. Venturi, S. Campagna and S. Serroni, Chem. Rev., 1996, 96, 759.

84 S. Valkama, O. Lehtonen, K. Lappalainen, H. Kosonen, P. Castro, T. Repo, M. Torkkeli, R. Serimaa, G. ten Brinke, M. Leskelä and O. Ikkala, Macromol. Rapid Commun., 2003, 24, 556.

85 D. G. Kurth, P. Lehmann and M. Schütte, Proc. Natl. Acad. Sci. U. S. A., 2000, 97, 5704.

86 D. G. Kurth, A. Meister, A. F. Thünemann and G. Förster, Langmuir, 2003, 19, 4055.

87 Y. Bodenthin, U. Pietsch, J. Grenzer, T. Geue, H. Möhwald and D. G. Kurth, J. Phys. Chem. B, 2005, 109, 12795.

88 Y. Bodenthin, U. Pietsch, H. Möhwald and D. G. Kurth, J. Am. Chem. Soc., 2005, 127, 3110 